\title{
Characterization of Dynamics and Power Handling of RF MEMS Using Vector Measurement Techniques
}

\author{
David Girbau, Antonio Lázaro, and Lluís Pradell, Member, IEEE
}

\begin{abstract}
This paper proposes a new method to measure dynamics and power handling of RF microelectromechanical systems (MEMS) devices based on a mobile membrane. The method uses in-phase/quadrature demodulation of an RF signal proportional to the reflection coefficient of the measured device, which contains information of its mechanical properties, such as actuation and release times and instantaneous position of the mobile membrane. Both one-port (capacitors) and two-port devices (switches and extended tuning-range capacitors) can be measured. Its main advantage is the capability of obtaining information from both magnitude and phase variations of the device reflection coefficient to characterize its dynamics and power handling. It is shown that detecting phase is advantageous in high quality factor capacitors, where the magnitude of the reflection coefficient is nearly constant for any position of the mobile membrane. Open-short-load calibration of the system is provided in order to obtain absolute measurements, which are necessary for power-handling characterization. The performances of the proposed method are demonstrated by comparison to systems based on detection of the magnitude of the reflection coefficient. A MEMS capacitor is characterized in terms of dynamics-actuation and release times, and mechanical resonance frequency-and in terms of power handling-membrane instantaneous position and phase and tuning range variation.
\end{abstract}

Index Terms-Dynamics, in-phase/quadrature (I/Q) demodulator, microwave microelectromechanical systems (MEMS) variable capacitor, MEMS switch, self-actuation.

\section{INTRODUCTION}

$\mathbf{M}$ ICROWAVE microelectromechanical systems (MEMS) is one of the modern technologies that has generated deep expectations to obtain high-performance devices, in addition to the capacity of integration and the cost, weight, and size reduction that it implies. However, there are a number of challenges and issues still to be solved, not only from the technological point-of-view and design concept, but also from the point-of-view of MEMS characterization. This paper will address the measurement of dynamics and power handling of RF MEMS.

Measurement of dynamics-actuation and release times, damping, and mechanical resonance frequency - is mandatory to have MEMS fully characterized, as this is one of the most important limitations of these devices due to their intrinsic mechanical principle of operation. These measurements can be done with an excellent resolution using optical techniques

Manuscript received April 21, 2004; revised June 29, 2004. This work was supported by the Spanish Government under Grant TIC2000-0144-P4-02 and Grant ESP2002-04 141-C03-02 (Ministerio de Ciencia y Tecnología).

The authors are with the Department of Signal Theory and Communications, Universitat Politècnica de Catalunya, 08034 Barcelona, Spain (e-mail: pradell@tsc.upc.es).

Digital Object Identifier 10.1109/TMTT.2004.837198
[1], but this leads to a very expensive measurement setup and to the impossibility of measuring packaged devices. Setups for measuring the dynamics of MEMS switches using microwave measurement techniques based on power detection have already been reported [2], [3].

On the other hand, power handling is the capability of a MEMS device to support RF power without changing its performance. Changes induced by RF power define an unwanted behavior that can be improved by design, but not completely overcome. Setups for measurement of power handling based on power detection have been reported up to date [4], [5].

This paper proposes a new method to measure dynamics and power handling of RF MEMS devices using instrumentation typically available in $\mathrm{RF} /$ microwave laboratories, and demonstrates its suitability in measuring one-port devices. It is based on vector measurements (detecting the phase and magnitude of the device reflection coefficient) as an alternative to detecting magnitude only, as proposed in [6]. This is performed using an in-phase/quadrature (I/Q) demodulator to measure the instantaneous position and variations of the mobile membrane as a function of time. In MEMS capacitors, information of the phase excursion is very important in order to characterize dynamics and power handling because it is independent of the quality $(Q)$ factor, while the excursion in the reflection-coefficient magnitude decreases as the $Q$ factor of the measured devices increases. To this end, a reflectometer is used to obtain information of both the reference signal injected to the MEMS and a signal proportional to the reflection coefficient of the MEMS as a function of time. In this way, a calibration is done to obtain the corrected reflection-coefficient magnitude and phase measurements, which are related to power handling. For the measurement of dynamics, the instantaneous position of the membrane is not necessary, but only its relative variation as a function of time; hence, no calibration of the system is needed.

The method proposed has the advantage of permitting characterization of MEMS from both the device reflection-coefficient phase and magnitude excursions, i.e., the difference in the values they take from one membrane position to another. Therefore, it makes the measurement of dynamics and power handling possible independently of the MEMS device loss. This method will be demonstrated in a capacitor application, but its usefulness can be extrapolated to the characterization of dynamics and power handling of RF MEMS switches.

\section{DyNAMics AND Power HANDLING OF RF MEMS}

\section{A. Dynamics}

The dynamics of electrostatically actuated MEMS devices is determined by the physical properties and dimensions of the 
device and by environmental conditions. Actuation and release times are an important disadvantage of MEMS components compared to field-effect transistors (FETs) and p-i-n diodes, limiting the speed to values in the order of microseconds. The dynamics of motion of a suspended membrane can be predicted using the one-dimensional (1-D) nonlinear differential equation of a second-order system as follows:

$$
m \frac{d^{2} x}{d t^{2}}+\xi \frac{d x}{d t}+k x=F_{e}
$$

where $m$ is the mass of the mobile membrane, $\xi$ is the damping coefficient, $k$ is the total elasticity constant of the suspension in the direction of motion, and $F_{e}$ is the attractive electrostatic force due to the input actuation voltage. In RF MEMS devices, this movement can be seen as a variation in its $\mathrm{RF}$ impedance-hence, in the reflection coefficient—ruled by the mechanics of a second-order system. This is the principle for extracting low-frequency information from $\mathrm{RF} /$ microwave measurement techniques proposed in this paper.

\section{B. Power Handling}

The limitations in power-handling capability of RF MEMS devices come basically from two aspects: self-actuation and current density excess. Self-actuation is the attractive force that appears on a membrane due to the rms voltage of the RF signal [7], [8], causing the membrane to deflect in a manner similar to an applied dc bias. When an RF signal $V_{\mathrm{RF}}=V_{o} \cos (w t)$ is injected simultaneously to the dc-bias signal $V_{\mathrm{DC}}$, the effective voltage applied to the device is expressed by

$$
V=\sqrt{V_{\mathrm{DC}}^{2}+\frac{V_{0}^{2}}{2}}
$$

In (2), the high-frequency terms have been omitted as the membrane only responds to voltages whose frequency is under its mechanical resonance frequency. The resultant electrostatic force can be written as

$$
F_{e}=-\frac{1}{2} \frac{\partial C}{\partial x}\left(V_{\mathrm{DC}}^{2}+\frac{V_{o}^{2}}{2}\right)
$$

where $C$ is the RF capacity between plates and $x$ is the displacement of the mobile membrane. Two contributions can be deduced from (3). The first contribution, the expected one, is the attractive force caused by the dc voltage. The second contribution is the one known as self-actuation, an added attractive force that appears on a membrane due to the rms voltage of the incident RF power, generally unwanted (not in MEMS power detectors, which can be based on this principle).

On the other hand, an excess of RF current density results in ohmic heating of the membrane due to the Joule effect, causing the material to expand and the membrane to deflect. This behavior is related to skin effect [9], [10] since the higher the frequency, the lower the skin depth and the higher the undesired heating. Any RF MEMS device under high RF power conditions experiences the two effects, but depending on the design, one may take more importance than the other. A third related problem appears when the membrane is made of two or more materials, with different thermal expansion coefficients, describing a movement similar to that of a bimorph thermal actuator. In this way, power measurements must be done at the operating frequency of the device. Power handling can be improved by design [4], [11], but the undesired effects of RF power cannot be completely overcome; hence, power-handling limitations must always be characterized in functional RF MEMS devices.

\section{Phase Versus Magnitude Measurement}

Measurement of dynamics has already been done in RF MEMS switches, where there is an important variation in the magnitude of the reflection/transmission coefficients between the two states, by recording the change in power transmitted through the switch using a power detector [3], or by detecting the variation of the magnitude of the reflection or transmission coefficients [2]. However, in MEMS capacitors, the device that is the subject of study in this paper, the higher the $Q$, the smaller the difference in the magnitude of the reflection coefficient between the two capacity states. Expressions (4) and (5) show the magnitude and phase of the reflection coefficient of a MEMS capacitor, respectively,

$$
\begin{aligned}
& \left|\Gamma_{\text {MEMS }}\right| \\
& \quad=\sqrt{\left(\frac{Y_{0}^{2}-G^{2}-\omega^{2} C^{2}}{\left(Y_{0}+G\right)^{2}+\omega^{2} C^{2}}\right)^{2}+\left(\frac{2 \omega C Y_{0}}{\left(Y_{0}+G\right)^{2}+\omega^{2} C^{2}}\right)^{2}} \\
& \quad \angle \Gamma_{\text {MEMS }} \\
& \quad=\arctan \frac{\left(-2 \omega C Y_{0}\right)}{Y_{0}^{2}-G^{2}-\omega^{2} C^{2}}
\end{aligned}
$$

where $Y_{0}$ is the reference admittance, $G=1 / R$ is the parallel conductance, $C$ is the capacity, and $\omega$ is the angular frequency in the parallel admittance model shown in Fig. 1(a). These equations show the above-mentioned nearly constant behavior of the reflection coefficient magnitude for high- $Q$ capacitors, where $G$ tends to zero, while it can be appreciated that the phase is basically independent of the losses in high- $Q$ capacitors. This is shown in Fig. 1, where both magnitude [see Fig. 1(b)] and phase [see Fig. 1(c)] of the reflection coefficient of a MEMS variable capacitor (the one which will be characterized in Section V, shown in Fig. 3) are plotted versus its capacity, sweeping the values of the parallel loss resistance $R$. It is clear that while the excursion in the magnitude of the reflection coefficient between two capacity states decrease when the $Q$ factor is increased, the phase excursion remains nearly constant.

It follows that an alternative to transmitted power detection and reflection-coefficient magnitude detection is to characterize the dynamics and power handling of MEMS capacitors from detection of the device reflection coefficient phase, as proposed in this paper. In addition, MEMS switches can also take an advantage of this system since small variations of a mobile membrane can be easily displayed. 


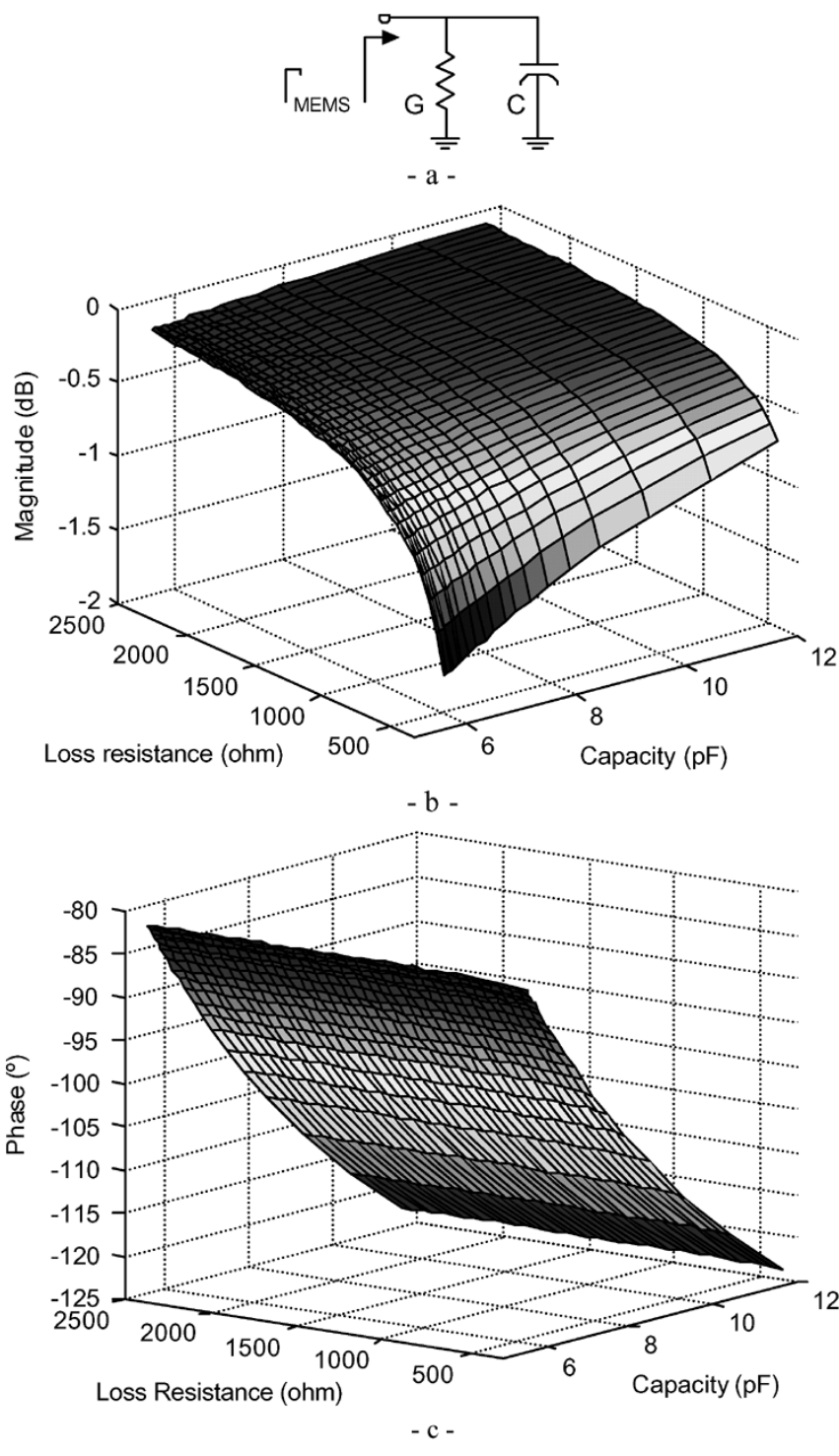

Fig. 1. (a) Parallel admittance model of the MEMS capacitor. (b) Reflection-coefficient magnitude and (c) phase excursions between two biasing (capacity) states versus loss resistance of the capacitor of Fig. 3 .

\section{Measurement Method}

The method proposed is based on the detection of a RF signal proportional to the reflection coefficient of the MEMS device. The devices are biased between two states and, simultaneously, an RF signal is injected. In this way, a time-dependent RF impedance-hence, reflection coefficient—is defined, whose magnitude and phase are modulated in amplitude according to the dynamics imposed by the mechanical movement of the membrane. Although the reflection coefficient is defined strictly for a steady-state sinusoid, it can be assumed that this is indeed the situation for this measurement since the RF carrier frequency is much larger than the low-frequency variations of the mechanical device. In the case of power-handling measurements, the MEMS device is in a steady-state situation, and both informations (corrected magnitude and phase) are required to extract the effects of RF power injection into the device.
Since both magnitude and phase of the reflection coefficient contain information of the mechanical movement, two options for the measurement arise.

\section{A. Scalar (Magnitude) Measurements}

A method to measure dynamics of MEMS from its reflection-coefficient magnitude has been presented in [6]. It is based on the peak detection of the amplitude-modulated magnitude of an RF signal proportional to the device reflection coefficient. It can be used both for testing capacitors and switches. A sample of the MEMS device reflection coefficient as a function of time, which contains the information of dynamics, is extracted using a directional coupler. Downconversion and detection at IF makes characterization of MEMS devices working at very different frequencies possible. The information is processed and displayed at an oscilloscope. Using the scalar system, the dynamics of low- $Q$ MEMS variable capacitors have been measured [6]. However, a limitation is the impossibility of detecting small reflection coefficient magnitude variations in high- $Q$ capacitors, as shown in (4) and Fig. 1(b).

\section{B. Vector (Magnitude and Phase) Measurements}

The phase transitions between two impedance states are independent of the device loss, and this makes small changes in the mobile membrane (and, hence, in the reflection coefficient) measurable. The experimental setup to measure the reflection coefficient phase and phase transitions, shown in Fig. 2, has been implemented to demonstrate the vector measurement concept proposed. A dc source and a square-shape function generator are simultaneously coupled to the device-under-test (DUT). They generate the bias voltage $\left(V_{\text {bias }}\right)$ injected to the MEMS device making it switch between the two states-between ON and OFF states in switches and, normally, between nominal and maximum capacity in capacitors. The function generator has time transitions in the order of nanoseconds. The bias signal can be applied to port $1\left(V_{\mathrm{bias}(1)}\right)$ and port $2\left(V_{\mathrm{bias}(2)}\right)$ to test both reflection (one- and two-port extended tuning range capacitors [12], [13]) and transmission devices (switches). In this way, the device switches between two impedance states. An RF tone is simultaneously injected to the MEMS through a reflectometer (two directional couplers or a dual directional coupler). This RF tone passes through a power amplifier and an attenuator to obtain enough power to actuate the device and sweep the power, respectively.

Hence, the magnitude and phase of the RF signal coupled at point $A$ of the measurement system (see Fig. 2), proportional to the reflection coefficient of the MEMS device, are amplitude modulated in time by the mechanical variations of the mobile membrane. While the measurements of interest are in terms of magnitude/phase variations as a function of time-this is the case of dynamics (actuation and release times), no calibration of the system is required. If a calibration is done, more information will be added to the time transitions, as, for example, the instantaneous position of the membrane.

However, when measuring power handling, corrected measurements are required. In this way, this effect cannot only be displayed, but also quantified, obtaining the instantaneous phase 


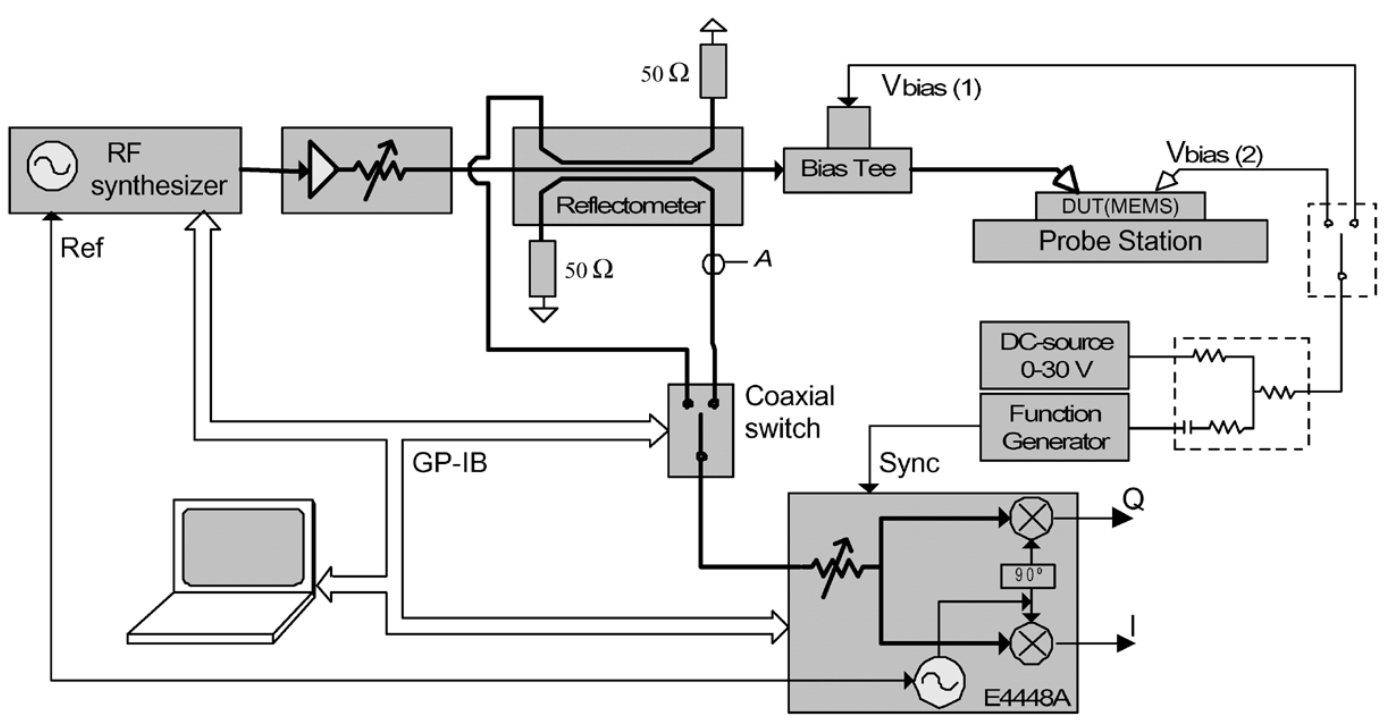

Fig. 2. Block diagram of the experimental setup used to implement the MEMS dynamics/power-handling vector measurement method proposed. Reflectometer directivity: $36 \mathrm{~dB}$. Coupling: $20 \mathrm{~dB}$ at $500 \mathrm{MHz}$.

and magnitude of the reflection coefficient, and making possible the determination of the capacity and position of the membrane as a function of the RF power. This solution imposes the need for calibration of the measurement system to extract the information of the MEMS device from its raw measurements. To this end, a sample of the incident wave is obtained from the reflectometer. Since a single measurement channel is used, a coaxial switch is placed to select the two outputs of the reflectometer and to implement open-short-load (OSL) calibration.

The measures of the incident and reflected waves to the device are demodulated using an I/Q demodulator (in our case, the Agilent E4448A Spectrum Analyzer). The I and Q channels are processed to obtain the information of the instantaneous magnitude and phase by applying the correction coefficients obtained from calibration. In this way, dynamics (and its dependence on RF power) and power handling can be characterized both from magnitude (in the case of enough change) and phase, as proposed in this paper. Synchronization of the I/Q demodulator to the biasing function generator is essential to obtain synchronous transitions between all measurements.

\section{OSL Calibration}

When measuring the effects of power on the position of the membrane, on its dynamics or on its tuning range, the information of the instantaneous corrected values of the reflection-coefficient phase and magnitude is mandatory, needing calibration. The well-known OSL reflection calibration technique is applied to this end, placing three known loads at the on-wafer plane for every RF power to be applied to the MEMS device, and solving the following three-equation system:

$$
\Gamma_{L, i} \cdot a+b-\Gamma_{L, i} \cdot \Gamma_{m, i} \cdot c=\Gamma_{m, i}, \quad \text { for } i=1,2,3
$$

where $a, b$, and $c$ are three unknowns from which the system error parameters can be determined, $\Gamma_{m, i}$ are the measured values of the reflection coefficients corresponding to calibration standards, and $\Gamma_{L, i}$ are known from the models provided for the on-wafer calkit standards $\left(\Gamma_{L, i}=1,-1,0\right.$ for an ideal case).
Once $a, b$, and $c$ are known, the reflection coefficient of the MEMS device $\Gamma_{\text {MEMS }}$ is obtained from its measured reflection coefficient $\Gamma_{m}$ (raw measurement)

$$
\Gamma_{\mathrm{MEMS}}=\frac{b-\Gamma_{m}}{c \cdot \Gamma_{m}-a} .
$$

\section{EXPERIMENTAL RESULTS}

The on-wafer MEMS capacitor measured to validate the proposed method is the one whose photograph, vertical cross section, and measured tuning range are shown in Fig. 3. Details of its design can be found in [12].

This is an electrostatically actuated capacitor with an extended tuning range of $7.27: 1$ at $500 \mathrm{MHz}$ with respect to the nominal capacity. The tuning range is extended by separating the actuation and capacity-testing electrodes [12]. This topology, along with the technological process [14]—one plate of the capacitor made of polysilicon and the other made of polysilicon plus gold-led to a low- $Q$ factor $(Q=11.4$ at $500 \mathrm{MHz}$ ). A low- $Q$ factor means that a fraction of the incident power will come into the device, as the power delivered to the load is proportional to $1-|\Gamma|$. This feature has been used to demonstrate the MEMS power-handling limitations of the device and the suitability of the measurement method proposed without requiring excessive power $(1 \mathrm{~W})$. Measurements have been done by switching the input dc voltage to the capacitor between $15-38 \mathrm{~V}$.

\section{A. Calibration Results}

An on-wafer 40-ps delay-line standard (not used in the calibration) has been measured at $500 \mathrm{MHz}$ in order to verify the correctness of the calibration and determine the residual errors. Fig. 4 shows the measurement of the magnitude of the reflection coefficient and the delay of the 40-ps line for the five powers that will be injected to the device $(7.6,10.9,14,17.2$, and $20.2 \mathrm{dBm})$. The error is less than $0.015 \mathrm{~dB}$ (magnitude) and $1.5 \mathrm{ps}\left(0.3^{\circ}\right.$ in-phase). 


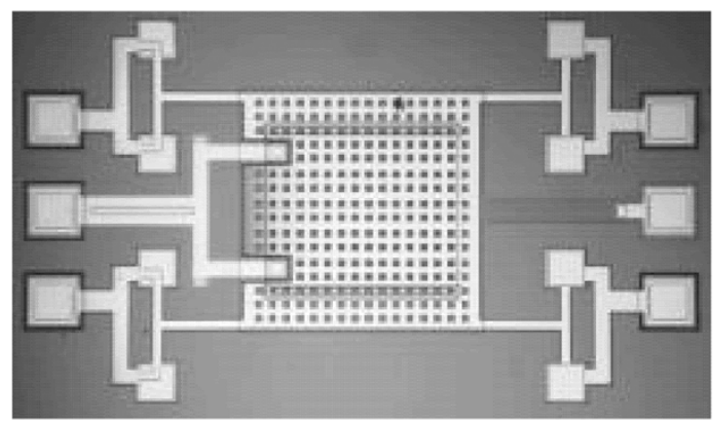

$-a-$

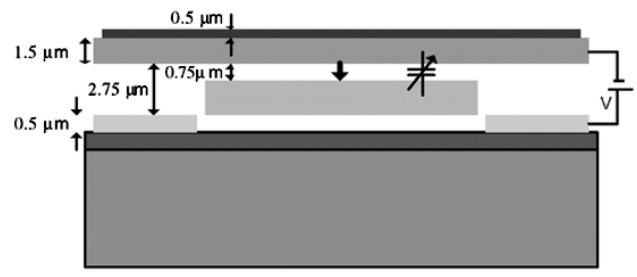

$-b-$

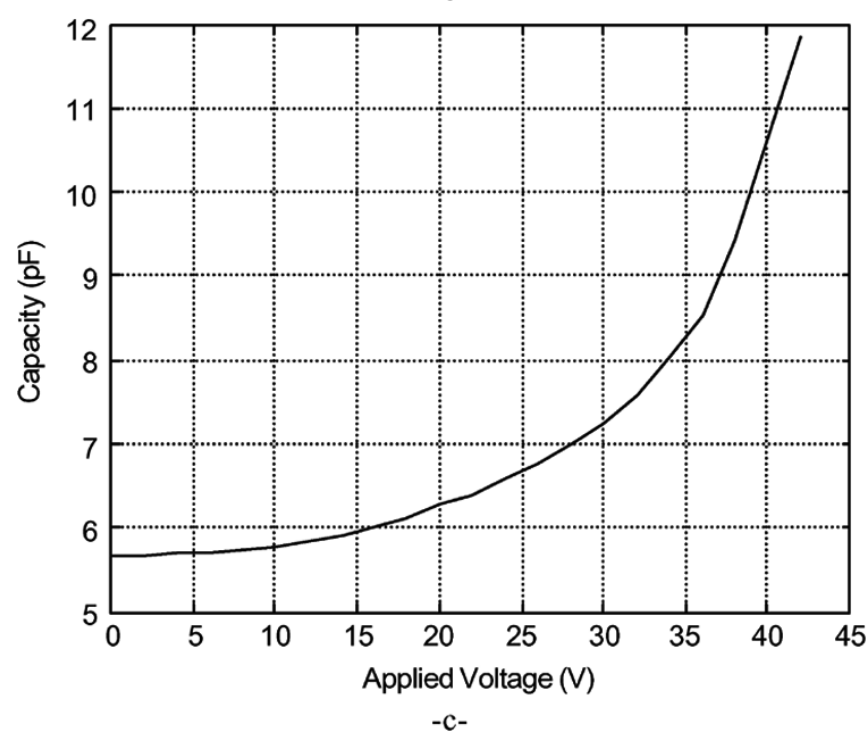

Fig. 3. (a) Electrostatically actuated capacitor with extended tuning range. (b) Vertical cross section. (c) Tuning range at $500 \mathrm{MHz}$ : 7.27:1 (with respect to the estimated nominal capacity: $0.986 \mathrm{pF}$ ) with actuation voltage of $0-42 \mathrm{~V}$. The device $Q$ is 11.4 at $500 \mathrm{MHz}$, from [6] and [12].

\section{B. Dynamics and Resonance Frequency Measurements}

Using the setup of Fig. 2, the dynamics of the capacitor of Fig. 3 has been measured. Fig. 5 shows the device measured actuation and release times. Table I compares actuation and release times extracted from Fig. 5 to the ones measured from the detected magnitude using the scalar system of [6]. The 10\%-90\% criteria has been taken. These measurements demonstrate the viability of measuring dynamics using phase information.

The device mechanical resonance frequency can be obtained by measuring both the reflection coefficient peak-to-peak magnitude or phase between the two states of the MEMS device as a function of the frequency of the biasing function generator using a sine-wave shape. Fig. 6 shows this measurement for the capacitor of Fig. 3 in an overdamped environment-open air. Under this situation, no peak of resonance frequency is observed, as the damping coefficient is smaller than 0.707, but it can be observed that, at $300 \mathrm{~Hz}$, the mobile membrane cannot completely
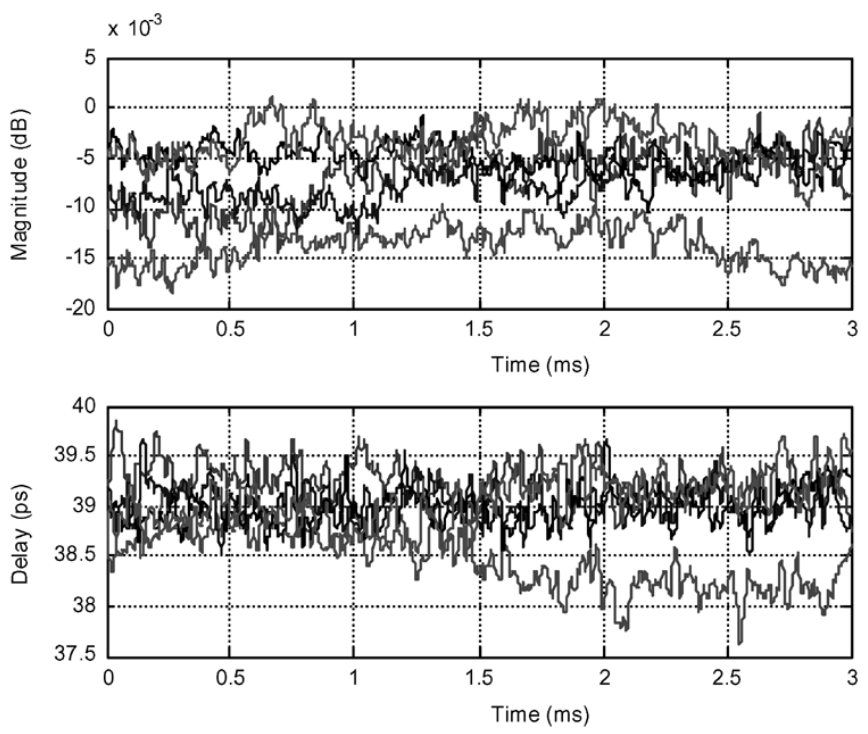

Fig. 4. Verification of the OSL calibration using a 40-ps delay line as a DUT — magnitude of the reflection coefficient and delay—for the input powers: $7.6,10.9,14.5,17.2$, and $20.2 \mathrm{dBm}$.
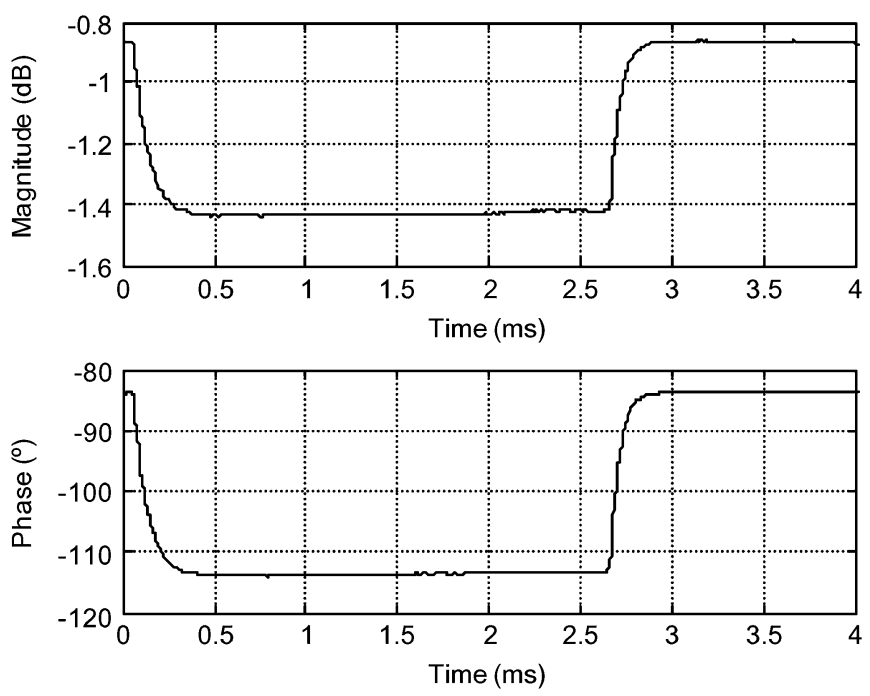

Fig. 5. Actuation and release times of the capacitor of Fig. 3 derived from the (upper) magnitude variation and (lower) phase variation.

TABLE I

ACTUATION/RELEASE TIMES

\begin{tabular}{lcc}
\hline \hline & Actuation Time & Release Time \\
\hline Magnitude measurement & $160.8 \mu \mathrm{s}$ & $109.7 \mu \mathrm{s}$ \\
Phase measurement & $159.3 \mu \mathrm{s}$ & $110.5 \mu \mathrm{s}$ \\
Measurement using system in [6] & $158 \mu \mathrm{s}$ & $115 \mu \mathrm{s}$ \\
\hline \hline
\end{tabular}

follow the input signal any more. Hence, this is the limit of the actuation frequency for this particular device under the above viscosity conditions.

\section{Power-Handling Measurements}

Using the measurements of the corrected reflection-coefficient phase, the power handling of the capacitor of Fig. 3 is characterized. Fig. 7 shows the effect of power on the position of the mobile membrane without applying dc biasing to the 


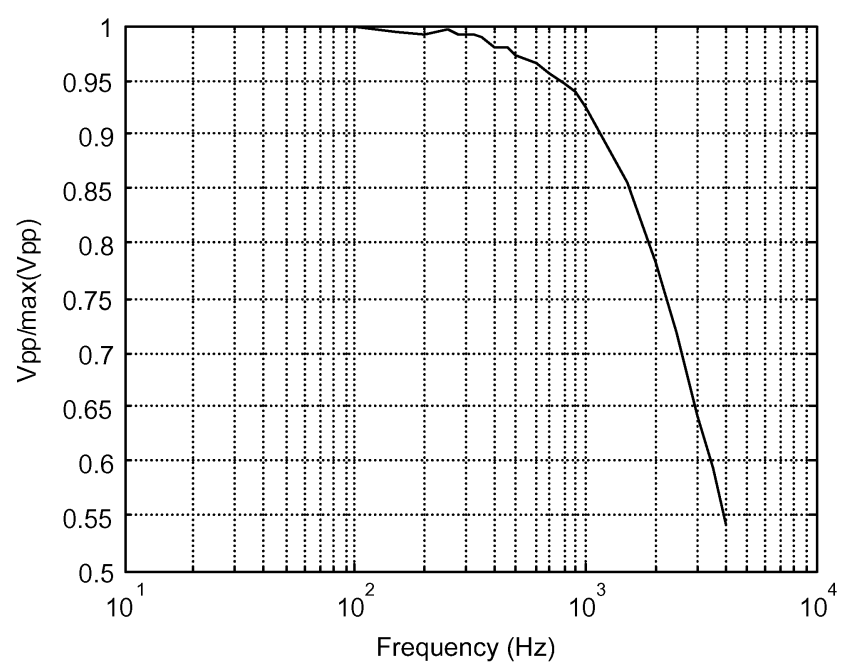

Fig. 6. Measurement of the behavior of the capacitor of Fig. 3 as a function of the frequency of the actuation signal under overdamped environmental conditions.

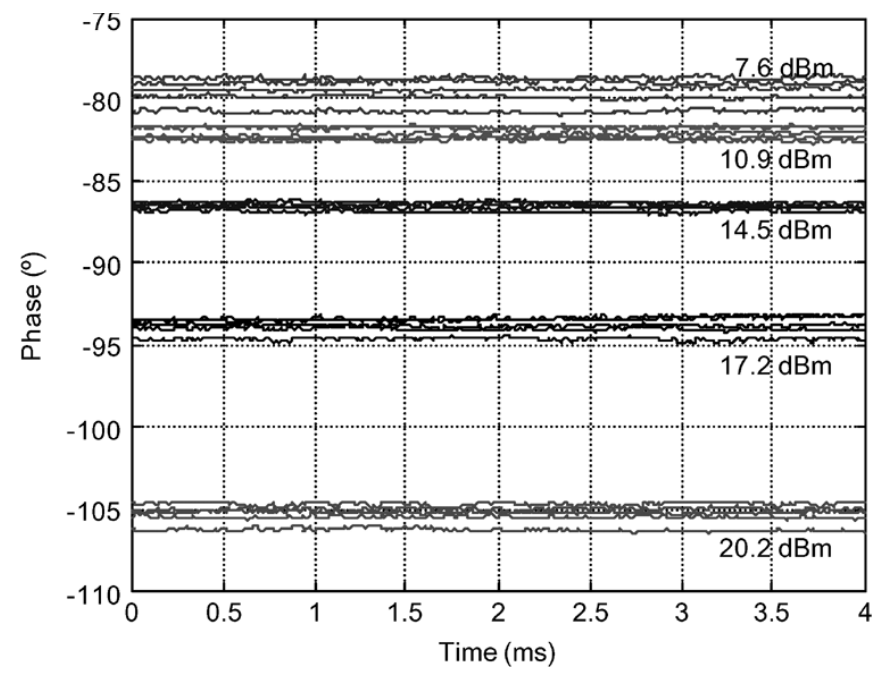

Fig. 7. Self-actuation due to RF power of the capacitor of Fig. 3. The capacity values for each RF power are: $5.67(7.6 \mathrm{dBm}), 5.71(10.9 \mathrm{dBm}), 6.12$ $(14.5 \mathrm{dBm}), 6.9(17.2 \mathrm{dBm})$, and $8.35 \mathrm{pF}(20.2 \mathrm{dBm})$.

device for five RF powers. The same measurement has been repeated five times to show the repeatability of the measurement system. It can be observed that the effect of RF power on the MEMS capacitor is to increase the total capacity from $5.66 \mathrm{pF}(7.6 \mathrm{dBm})$ to $8.34 \mathrm{pF}(20.2 \mathrm{dBm})$ as a result of a downward movement of the mobile membrane. The values of capacity are directly obtained from the I/Q measurements using the proposed system or from the small-signal measurements using a vector network analyzer (VNA) (see Fig. 3).

To draw a distinction between the contribution of self-switching and excess current density in a given power-handling measurement is a difficult task. However, in the case of the capacitor of Fig. 3, it is observed that one of the suspensions of the top plate is blown for an input RF power of $+25 \mathrm{dBm}$. This result indicates that an excess current density could have an important contribution to the displacement of this membrane due to the RF power.

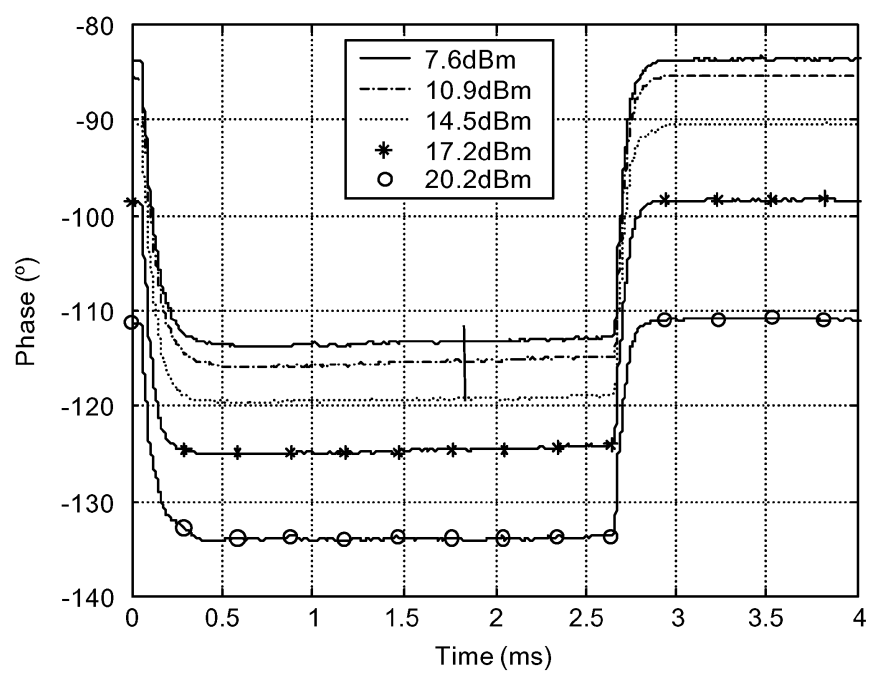

Fig. 8. Effect of RF power on dynamics and tuning range of the capacitor of Fig. 3, switching between two states (15-38 V).
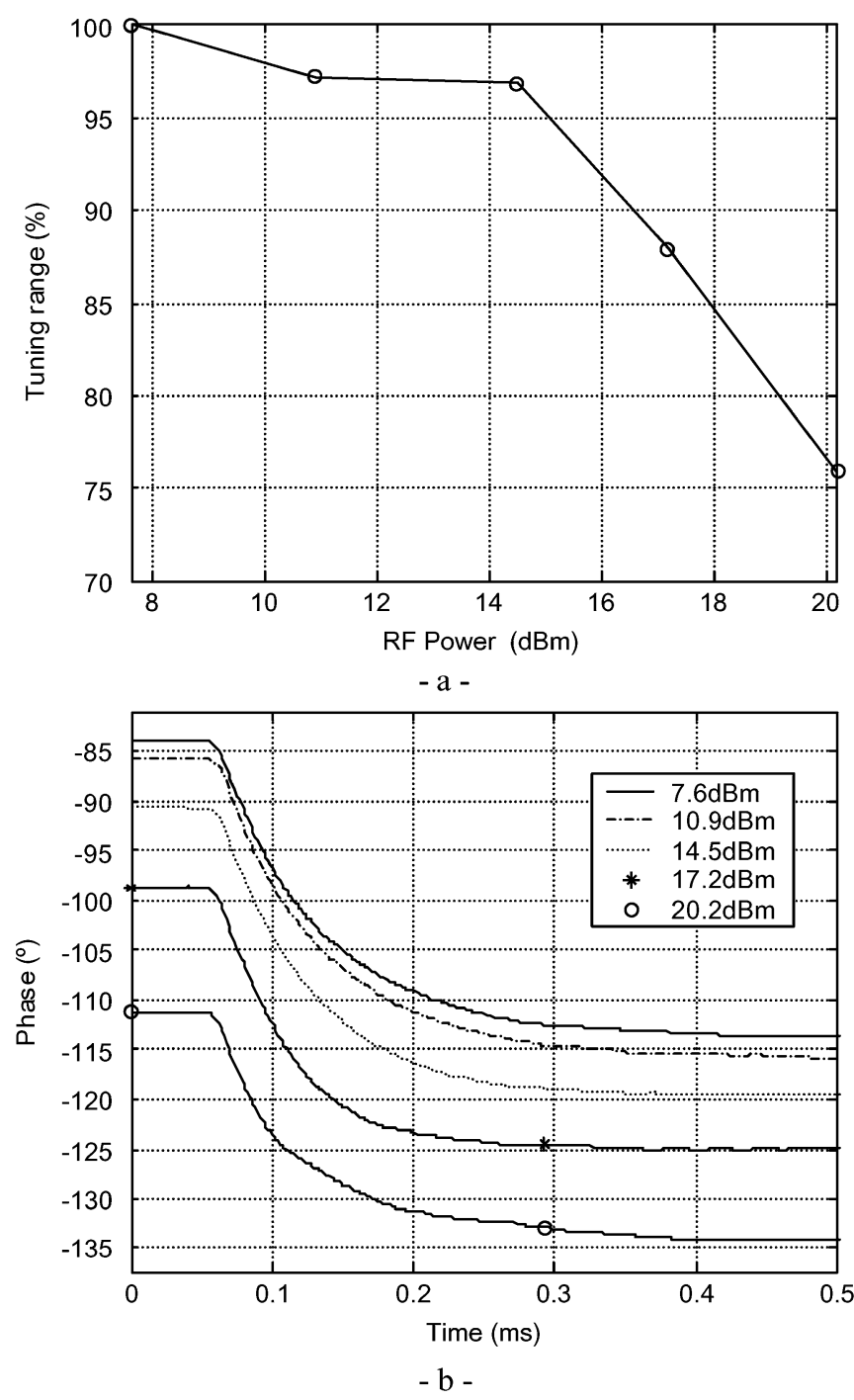

Fig. 9. (a) Effect of RF power on the tuning range of the capacitor of Fig. 3. (b) Effects of RF power on the dynamics of the capacitor in the actuation transition. 
Another important effect of the RF power is the variation of the tuning range and dynamics, as it is experimentally demonstrated in Fig. 8 for the capacitor of Fig. 3. A simultaneous switching of the capacitor (biasing dc voltage from 15 to $38 \mathrm{~V}$ ) and injection of RF power is provided for five RF powers (7.6, $10.9,14.5,17.2$, and $20.2 \mathrm{dBm}$ ). The result is a modification of dynamics - the actuation time tends to decrease - of the nominal capacity-increased due to the bending effect on the mobile membrane, which approaches the two plates-and of the tuning range-reduction of the peak-to-peak detected phase-as a function of the injected RF power. These modifications of the dynamics and tuning range are shown in detail in Fig. 9, where a plot of the capacitor tuning range versus input $\mathrm{RF}$ power is provided, indicating a $24 \%$ tuning range decrease at an RF power of $20.2 \mathrm{dBm}$.

\section{CONCLUSION}

In this paper, the advantages of using detection of the reflection-coefficient phase to characterize dynamics and power handling of RF MEMS devices has been presented. The necessity of using this method in high- $Q$ capacitors has been discussed and the advantages of using it in switches have been pointed out. An experimental setup based on I/Q demodulation has been implemented and used to characterize dynamics and power handling of an on-wafer extended tuning-range MEMS capacitor.

Measurements of dynamics of the MEMS device have been compared using a scalar (magnitude) measurement system and a vector measurement system (magnitude and phase) to demonstrate the suitability of phase measurements. An OSL calibration is performed in order to extract the systematic errors of the measurement system. Power-handling measurements, also based on reflection-coefficient phase detection, have been done, showing the effects of RF power-self-actuation and excess current density. The RF power causes a movement of the suspended membrane of the measured capacitor, which translates into an increment of the nominal capacity, a reduction of tuning range, and a modification of dynamics (actuation and release times).

\section{REFERENCES}

[1] E. Lawrence and K. Speller, "MEMS characterization using laser Doppler vibrometry," Polytec, Appl. MEMS.

[2] S. Mellé, F. Flourens, D. Dubuc, K. Grenier, P. Pons, J. L. Muraro, O. Vendier, and R. Plana, "Early degradation behavior in parallel MEMS switches," in 4th MEMS for Millimeterwave Communications Workshop, July 2003, pp. F33-F-36.

[3] D. Peroulis, S. P. Pacheco, K. Sarabandi, and L. P. B. Katehi, "Electromechanical considerations in developing low-voltage RF MEMS switches," IEEE Trans. Microwave Theory Tech., vol. 51, pp. 259-270, Jan. 2003.

[4] D. Peroulis, S. P. Pacheco, and L. P. B. Katehi, "RF MEMS switches with enhanced power-handling capabilities," IEEE Trans. Microwave Theory Tech., vol. 52, pp. 59-68, Jan. 2004.

[5] B. Pillans, J. Kleber, C. Goldsmith, and M. Eberly, "RF power handling of capacitive RF MEMS devices," in IEEE MTT-S Int. Microwave Symp. Dig., vol. 1, June 2002, pp. 329-332.

[6] D. Girbau, A. Lázaro, and L. Pradell, "Characterization of dynamics in on-wafer RF MEMS variable capacitors using RF measurement techniques," in 63rd ARFTG Microwave Measurement Conf. Tech. Dig., June 2004, pp. 117-123.

[7] K. M. Strohm, B. Schauwecker, D. Pilz, W. Simon, and J.-F. Luy, "RF-MEMS switching concepts for high power applications," in Silicon Monolithic Integrated Circuits in RF Systems Topical Meeting, Sept. 2001, pp. 42-46.
[8] A. Cruau, P. Nicole, G. Lissorgues, and C.-M. Tassetti, "Influence of RF signal power on tunable MEMS capacitors," in Proc. 33rd Eur. Microwave Conf., vol. 2, Oct. 2003, pp. 663-666.

[9] W. Thiel, K. Tornquist, R. Reano, and L. P. B. Katehi, "A study of thermal effects in RF-MEM-switches using a time domain approach," in IEEE MTT-S Int. Microwave Symp. Dig., vol. 1, June 2002, pp. 235-238.

[10] B. D. Jensen, K. Saitou, J. L. Volakis, and K. Kurabayashi, "Impact of skin effect on thermal behavior of RF MEMS switches," presented at the 6th ASME-JSME Thermal Engineering Joint Conf., June 2003, Paper TED-AJ03-420.

[11] B. Ducarouge, E. Perret, F. Flourens, H. Aubert, J. W. Tao, X. Chauffleur, J. P. Fradin, D. Dubuc, K. Grenier, P. Pons, and R. Plana, "Design of MEMS-based microwave and millimeterwave switches for high power measurements," presented at the Int. Semiconductor Conf., vol. 1, Sept. 2003.

[12] D. Girbau, A. Lázaro, and L. Pradell, "Extended tuning range RF MEMS variable capacitors using electrostatic and electrothermal actuators," in Proc. SPIE Micromachining and Microfabrication, vol. 5344, Jan. 2004 , pp. $59-70$.

[13] J. Zou, C. Liu, J. Schutt-Aine, J. Chen, and S.-M. Kang, "Development of a wide tuning range MEMS tunable capacitor for wireless communication system," in Int. Electron Devices Meeting Tech. Dig., Dec. 2000, pp. 403-406.

[14] D. Koester, A. Cowen, R. Mahadevan, M. Stonefield, and B. Hardy, PolyMUMPS Design Handbook, Rev. 10.0. Durham, NC: Memscap, 2003.

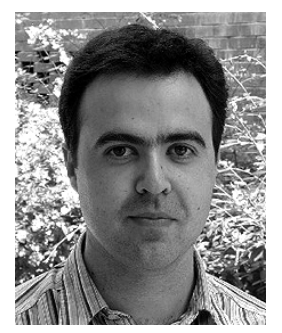

David Girbau received the Technical Engineer degree in telecommunications and Engineer in electronics degree from the Universitat Politècnica de Catalunya, Barcelona, Spain, in 1998 and 2002, respectively, and is currently working toward the Ph.D. degree at the Universitat Politècnica de Catalunya.

His research is currently focused on MEMS with an emphasis on their RF and microwave applications.

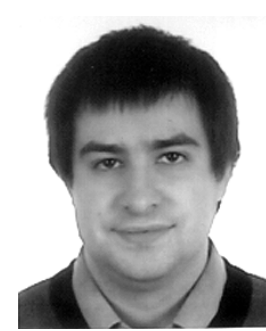

Antonio Lázaro was born in Lleida, Spain, in 1971 He received the M.S. and Ph.D. degrees in telecommunication engineering from the Universitat Politecnica de Catalunya (UPC), Barcelona, Spain, in 1994 and 1998, respectively.

He then joined the faculty of UPC, where he currently teaches a course on microwave circuits and antennas. His research interests are microwave device modeling, on-wafer noise measurements, monolithic microwave integrated circuits (MMICs), low phasenoise oscillators, MEMS, and microwave systems.

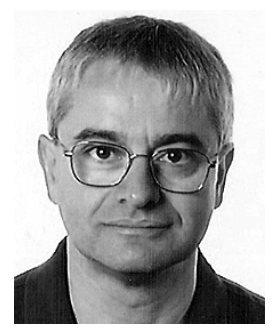

Lluís Pradell (M'04) was born in Barcelona, Catalunya, Spain, in 1956. He received the Telecommunication Engineering degree and Dr. degree in telecommunication engineering from the Universitat Politècnica de Catalunya (UPC), Barcelona, Spain, in 1981 and 1989, respectively.

From 1981 to 1985, he was an RF and Microwave System Design Engineer with Mier-Allende, Barcelona, Spain. In 1985, he joined the faculty of UPC, where, in 1990, he became an Associate Professor. Since 1985, he teaches courses on microwave circuits and antennas, and performs research on models for microwave active devices (MESFETs), high electron-mobility transistors (HEMTs), HBTs), multimodal models for guiding structures and transitions [microstrip, finline, slotline, coplanar waveguide (CPW)], on-wafer measurement techniques (network-analyzer calibration, noise parameters), development of microwave and millimeter-wave systems (low-noise amplifiers and point-to-multipoint broad-band communication systems), and RF and microwave MEMS in the $1-75-\mathrm{GHz}$ frequency range. 